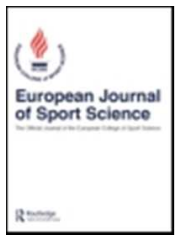

\title{
PHYSICAL ACTIVITY PROFILES AND SELECTED MUSCULAR FITNESS VARIABLES IN ENGLISH SCHOOLCHILDREN: A NORTH-SOUTH DIVIDE?
}

\begin{tabular}{|r|l|}
\hline Journal: & European Journal of Sports Science \\
\hline Manuscript ID & TEJS-2015-0719.R1 \\
\hline Manuscript Type: & Original Paper \\
\hline Keywords: & Assessment, Children, Fitness, Lifestyle, Performance \\
\hline \multicolumn{2}{|l}{} \\
\hline
\end{tabular}

\section{SCHOLARONE $^{\text {Wh }}$}

Manuscripts 
Abstract:

Introduction: The aim of the study was to compare and contrast habitual physical activity profiles and muscular fitness in schoolchildren from northern and southern regions of England.

Methods: Data were collected from two secondary schools in the North East (NE) of England. The study procedures followed methods employed by the East of England Healthy Hearts Study in 10-16 year old boys and girls based in the south east (SE) region of England and data were compared. Habitual physical activity (PAQ-A), vertical jump test (VJT), and hand-grip (HG) strength were assessed. We converted raw scores from all assessments to age- and sex-normalised z-scores.

Results: We recruited 597 children ( $58 \%$ boys) in the NE and compared findings to 597 age- and sex- matched boys and girls from the SE. Boys in the SE had significantly stronger HG scores, jumped higher, were more powerful (mean peak power: $2131 \mathrm{~W} \vee 1782 \mathrm{~W} ; P<0.0001$ ), and reported being more physically active (mean PAQ-A: $2.9 \vee 2.5 ; P<0.0001$ ) than their male counterparts in the NE. In girls, the opposite trend was evident. Girls from the NE of England had a higher HG score, jumped higher, and were more powerful (mean peak power: 2114W $\mathrm{V}$ $1839 \mathrm{~W} ; P<0.0001$ ) than their peers from the SE.

Conclusion: Regional variations in the habitual physical activity profiles and muscular fitness of schoolchildren from the SE and NE of England do exist. The systematic surveillance of children's physical activity and fitness profiles throughout England would help identify regional inequalities on a larger scale.

(Word Count: 250)

Keywords: vertical jump test; hand-group strength; habitual physical activity; boys; girls; regional differences and associations 


\section{Introduction}

It has been well established that whilst levels of childhood obesity have increased dramatically in Western societies over the past 25 years, levels of muscular fitness have declined significantly in young people. ${ }^{1,2}$ Muscular fitness is an important component of overall health status as well as a predictor of future health-related outcomes. ${ }^{3}$ English schoolchildren have shown a decrease in upper body muscular strength, measured by hand-grip strength (HGS) over the past decade ${ }^{1,2}$; a trend also reported in both Spain and Canada. ${ }^{4,5}$ Levels of lower body strength/power assessment via the vertical jump test (VJT) have also shown similar downward trends in recent years in young people. ${ }^{6,7}$

Whilst muscular fitness continue to decline in young people living in Western societies; it is perhaps not surprising to note that physical activity (PA) levels have also declined, especially in girls, over a similar time frame. ${ }^{8,9}$ This is particularly concerning given the strong association between low levels of PA and increased risk of cardiovascular disease and musculoskeletal ill-health. ${ }^{10,11}$ The accurate quantification of PA remains problematic; whilst objective measures such as accelerometry may provide a valid and reliable assessment of PA, such technology may be impractical in large-scale field-based settings. Self-reported PA inventories offer a frequently used alternative methodology despite the accepted limitations of subjective and questionable recall ability, especially in young people. ${ }^{12-14}$ The Physical Activity Questionnaire for Older Children (PAQ-A) has been used to develop normative and criterion-referenced PA values for UK children (boys and girls) aged between $10-16$ years. ${ }^{15}$

In the UK, data from the East of England Healthy Hearts Survey (EoEHHS) has provided much of our understanding of the current normative levels of physical activity and physical fitness in school-children ${ }^{1,2,6,15}$ There are data from other regional centres e.g. North West England, though this has focused mainly on cardiorespiratory fitness estimated using the multi-stage fitness test. ${ }^{16}$ One comparison of children's fitness profiles between NW and SW regions of England in small groups of boys and girls ( $\mathrm{n}<60$ participants per group) produced mixed results. Children in the SW of England had a lower body fat, and showed greater upper body strength (HG dynamometry) than children in the NW, whereas children 
from the NW region demonstrated greater lower body explosive strength. Differences in body fat may explain some of this variation but were not controlled for. Neither did the study assess or control for any indicator of physical activity. ${ }^{17}$

Evidence for lower muscular fitness and reduced habitual physical activity of English youth are worrying as both are associated with negative health outcomes. England has no framework to support the systematic surveillance of children's physical fitness. No comparable data exist for child fitness and it is unclear whether the data published from the EoEHHS ${ }^{1,2,6,15}$ are representative of young people's physical activity and fitness profiles across England or, if regional variations exist. Therefore, the aim of the study was to compare and contrast habitual physical activity profiles and selected strength and power variables in a sample of school-children from northern and southern regions of England.

\section{Methods}

\section{Sample}

The sample was initially drawn from two secondary schools in the East Riding of Yorkshire and was restricted to schoolchildren with a complete data set including age, height, weight and complete PAQ-A data. The study was approved by the Department of Sport, Health \& Exercise Science ethics committee at the University of Hull and conforms to the Declaration of Helsinki. ${ }^{18}$ Written, informed consent for pupils' participation was obtained from parents and each child gave verbal assent prior to participating in the assessment.

The EoEHHS was launched in 2007 and involved fitness testing 10-16 year-old children during PE classes at schools in the South and East of England including Suffolk, Essex and North London. After testing $>10000$ children in these areas, age- and sex-matched normative data for physical activity profiles, and cardiorespiratory and muscular fitness were published. ${ }^{1,2,6,15}$ Working with the Principal Investigator (GS) from the EoEHHS from the out-set, staff (LI) from a Higher Education Institution in the North East of England (NE) aimed to compare physical activity profiles and muscular performance of age- and sex-matched 
children from both regions using precisely the same methodologies. We compared the sample of children from the NE in relation to normative percentile data based on over ten thousand children from the EoEHHS in the SE region. Data from the NE region was collected in 2014 by one investigator (AS) who, after guidance from GS, replicated the methodological approach using identical equipment as the EoEHHS investigators. The two schools selected for inclusion in the NE were defined as 1) urban; and 2) rural based on geographical location following definitions provided by the rural-urban classification in 2011 by the UK government. ${ }^{19}$ Similarly, the two schools in the SE region, selected as direct comparators were also classified as 1) urban and 2) rural and were selected based on similar lower layer super output areas (LSOAs; details below).

\section{Protocol}

Schoolchildren undertook all assessments during regularly scheduled physical education $(\mathrm{PE})$ lessons. Stature $(\mathrm{cm})$ and body mass $(\mathrm{kg})$ were measured without shoes and in shorts and t-shirts) were recorded to the nearest $0.1 \mathrm{~kg}$ and $1 \mathrm{~mm}$ respectively (Seca, Hamburg, Germany). Body mass index (BMI) $\left(\mathrm{kg} \cdot \mathrm{m}^{-2}\right)$ calculated and classified according to International Obesity Task Force (IOTF) criteria. ${ }^{20}$ Area level deprivation (Indices of Multiple Deprivation 2007 [IMD2007]) was determined based on home postal codes. ${ }^{21}$ England is divided into 32482 LSOAs; each covering an average of $4 \mathrm{~km}^{2}$ with a mean population of 1500 . Each participant provided their home postcode from which we determined the LSOA in which they resided. LSOAs are classified as either urban (output area lies within settlements with a population of $>10000$ ) or rural which includes town and fringe areas, villages or isolated dwellings. Deprivation was evaluated by calculating the English Index of Multiple Deprivation (IMD) for each LSOA. The 2007 English Indices of Multiple Deprivation $^{21}$ provides a powerful tool for the identification and analysis of deprived areas across England by combining 37 separately weighted indicators into a single deprivation score. A higher IMD score is indicative of a more deprived LSOA. 


\section{Physical Activity Questionnaire (PAQ-A)}

The validation of the PAQ-A questionnaire has been described elsewhere. ${ }^{15}$ Briefly, the self-administered, 7-day recall questionnaire comprises nine items and identifies information on participation in different types of sports and activities, effort produced during PE classes, and physical activity during lunch, after school, evening and at the weekend during the past 7 days. Each item is scored on a Likert scale between 1 (low PA) and 5 (very high PA) and the overall score denotes the PAQ score.

\section{Vertical Jump Test (VJT)}

The VJT followed the protocol used by Taylor and colleagues. ${ }^{6}$ Children wore appropriate sports footwear for the VJT. Initially, the researcher demonstrated the jump technique (counter-movement with arm swings) and each child practised until they met the required criteria. The jump began from a standing position, with the feet and leg vertically aligned at approximately $180^{\circ}$. When the countermovement was performed, the knees flexed to approximately $90^{\circ}$ before rapid extension and take-off. Each child held a piece of chalk in the dominant hand and marked the wall where they reached the apex of the jump. Landing required knee angle to be extended to approximately $180^{\circ}$. If the criteria were not met, the jump was performed again. We measured in centimetres the difference between standing height with arm extended vertically and the distance reached at the apex of the jump. We recorded this value as the jump height achieved. Each child was permitted two jumps using the correct technique and the best jump height was recorded. Following the Taylor and co-workers ${ }^{6}$ protocol, we predicted peak power output using the following equation developed by Sayers and colleagues ${ }^{22}$ :

Peak power $(\mathrm{W})=60.7 \times($ jump height, $\mathrm{cm})+45.3 \times$ (body mass, $\mathrm{kg})-2055$

\section{Handgrip Strength (HG)}

The HGS dynamometer has been shown to be a valid and reliable method of strength assessment in young people. ${ }^{23,24}$ The HG protocol has been provided 
elsewhere. ${ }^{1}$ In brief, each child was given a brief demonstration and verbal instructions in the correct use of the handgrip dynamometer (Takei Scientific Instruments Co. Ltd, Tokyo, Japan). For each child, the device was adjusted in order to accommodate differences in hand size. The test was conducted in the standing position with the wrist in the neutral position and the elbow extended. ${ }^{25}$ The arm position of the dominant hand was allowed to move between $180^{\circ}$ of flexion to $0^{\circ}$. Participants were given verbal encouragement to apply maximal effort in the range of $0^{\circ}$ to $90^{\circ}$ of flexion and 'squeeze as hard as possible' for a minimum of two seconds, on two separate occasions (over a five minute period). The highest score recorded over the two trials was taken as the as peak grip strength $(\mathrm{kg})$.

\section{Data Treatment}

We converted raw scores from all tests and assessments to age- and sexnormalised z-scores. This process allows the pooling of data from pupils of different ages and allows direct comparison of samples with different mean age and samples with expected differences in raw scores (e.g. boys and girls). The use of z-scores also corrects for skewness and kurtosis allowing the application of parametric analyses. The PAQ-A z-scores were calculated based on normative English data. ${ }^{26}$ Handgrip strength was also expressed as a z-score using English (EoEHHS) reference data. ${ }^{1}$ Jump height and peak power expressed as z-score based on English reference. ${ }^{6}$ As handgrip strength and peak power are both associated with body mass we also scaled raw scores on these tests for body mass to create relative scores (handgrip, $\mathrm{kg} \cdot \mathrm{kg}^{-1}$; peak power, $\mathrm{W} \cdot \mathrm{kg}^{-1}$ ). To assess whether one was a suitable exponent we correlated relative measures with body mass to ensure no significant correlation remained.

\section{Statistical Analysis}

We assessed the differences in physical activity profiles and fitness scores between regions (NE and SE of England) using independent samples t-tests (Table 2). To determine the association between region and outcome measures, we first coded 
region as a dummy variable $\mathrm{SE}=0, \mathrm{NE}=1$. We then performed hierarchical linear regression analysis with region forced into the equation. SPSS version 22 (IBM, NY, USA) was used to analyse the data. An alpha level of $P<0.05$ was accepted as significant.

\section{Results}

In the NE region of England, we recruited 597 children (58\% boys) aged between 11.0-15.9 years from two secondary schools. These data were compared to ageand sex- matched boys and girls from the SE region $(n=597)$. Table I provides the raw scores in HGS, VJT and physical activity profiles for boys and girls across the two regions. Table II shows mean standardised values (SD) for anthropometric and performance characteristics, and physical activity profiles in the boys and girls in the NE and SE regions of England. Boys from the SE were taller $(z=0.43)$ and had a higher BMI ( $z=0.29 ; 95 \% \mathrm{Cl}: 0.05-0.52 ; \sim 8$ percentile points) than boys from the NE. More boys were classified as underweight (7.8\% SE $\vee 15.2 \%$ NE; $P<0.001)$ was more prevalent than being classified as obese (5.8\% SE v 7.2\% NE; $P=0.40)$. Girls from the NE weighed more than those from the SE $(53.6 \pm 15.6 \mathrm{~kg} \vee 50.9 \pm 12.3 \mathrm{~kg}$; $P=0.02$ ) but their BMI was lower (though not significantly; $19.9 \pm 4.4 \mathrm{~kg} \cdot \mathrm{m}^{-2} \vee 20.3 \pm$ $4.7 \mathrm{~kg} \cdot \mathrm{m}^{-2} ; \quad P=0.22$ ) as they were taller than expected for their age. Being classified as underweight was more prevalent than being classified as being obese in both cohorts, but was nearly twice as likely in girls from the NE compared to those from the SE (7.8\% SE v 15.2\% NE; $P<0.001)$.

Differences in standardised physical activity profiles and selected fitness scores between boys in the NE and SE are provided in Table III. Boys in the SE had significantly stronger HG scores (mean HG: $26.3 \vee 22.8 ; P=0.013$ ), jumped higher (mean VJT: $0.33 \mathrm{~m} \vee 0.28 \mathrm{~m}$; $P<0.0001$ ), were more powerful (mean peak power: $2131 \mathrm{~W} \vee 1782 \mathrm{~W} ; P<0.0001$ ), and reported being more physically active (mean PAQ-A: $2.9 \vee 2.5 ; P<0.0001)$ than their male counterparts in the NE. Boys from the SE performed near the expected level for age and sex in both HG and VJT, their 
values were significantly better than those recorded by boys in the NE whose performance was below expected values for HG $(z=-0.70)$ and VJT $(z=-0.60)$.

In girls, the opposite trend was evident. Girls from the NE of England had a higher HG score (mean HG score: $27.9 \vee 22.4 ; P=0.014$ ), jumped higher (mean VJT: 0.30 $\mathrm{m} \vee 0.28 \mathrm{~m} ; P=0.031$ ), and were more powerful (mean peak power: $2114 \mathrm{~W} \vee 1839$ W; $P<0.0001)$ than girls from the SE. Girls from the SE had HG strength at the expected level for their age but girls from the NE performed well above this level ( $z=0.80 \pm 1.52 ; \sim 93^{\text {rd }}$ percentile based on age). VJT height was above the values expected for relative age in both cohorts, but girls from the NE performed at the $70^{\text {th }}$ percentile $(z=0.53 \pm 1.53 ; 8$ percentile points higher than those from the $S E)$. Self-reported physical profiles were as expected for age; girls from the NE reported being less active $(z=-0.15,95 \% \mathrm{Cl}:-0.31-0.01 ; 6$ percentiles points lower $)$ than girls in the SE, though this was not statistically significant $(P=0.06)$.

We then identified the influence of anthropometric variables and performed linear regression analyses on absolute and normalised values for handgrip $(\mathrm{kg}, \mathrm{kg} / \mathrm{kg}$ body weight) and peak power output (W, W/kg). Table IV shows age-adjusted values followed by anthropometric values (BMI) identified as significant correlates (data not shown). We then adjusted these estimates for physical activity, and finally area level deprivation. Age, mass, and stature all predicted HG strength but location remained a significant factor $(B=3.9 \mathrm{Kg} ; 95 \% \mathrm{Cl}-4.70$ to -2.93$)$ in the lower values for boys from the NE region of England. The addition of area-level deprivation improved the prediction of HG strength. Boys from the SE were significantly more powerful than equivalent boys from the NE after adjusting for age and BMI. The addition of physical activity to the model attenuated the association slightly. Deprivation was negatively associated with peak power output but location remained a significant predictor in the fully-adjusted model with boys from the NE (448 W; $95 \% \mathrm{Cl}$ : -649 to $-247 \mathrm{~W})$. Relative peak power output $\left(\mathrm{kg} / \mathrm{kg} \cdot \mathrm{BW}^{-1}\right)$ was higher in boys from the SE after adjusting for age and BMI. PA was positively associated with relative peak power output and its addition to the model attenuated the contribution of location. In the fully-adjusted model, the effect of location was reduced but remained significant. 


\section{Discussion}

Our study is the first to show sex-specific regional variations in the muscular fitness and physical activity patterns between schoolchildren from the north (NE) and south (SE) of England. We found a regional role reversal between boys and girls. Compared with boys from the NE, those from the SE of England were significantly stronger and more powerful; they also reported higher levels of physical activity. Conversely girls from the NE of England were stronger and more powerful than girls from the SE of the country. Regional differences in muscular strength and power remained significant even after adjusting for variations in age, anthropometric measures, physical activity and area level deprivation.

The importance of assessing levels of physical activity or inactivity cannot be understated; previous studies have established a strong association between physical inactivity and an increased prevalence of overweight and obesity. ${ }^{27,28}$ Others have reported associations between overweight/obesity and sedentary behaviour, such as excessive screen time in yout. ${ }^{29,30}$ Boyle and colleagues ${ }^{31}$ reported that only $25 \%$ of children (11-15 years) from four English schools (2xNW; $\mathrm{x} 2 \mathrm{SW}$ ) engaged in 60 min daily moderate to vigorous physical activity (MVPA). The 2012 Heath Survey for England ${ }^{32}$ reported that $14 \%$ of boys and $8 \%$ of girls (13-15 years) met the recommendations for achieving at least 60 minutes of MVPA on seven days a week using a self-reported measurement tool. Further, $39 \%$ of boys and $45 \%$ of girls aged 5-15 years were classified as having low levels of physical activity ( $<30$ minutes of MVPA on each day, or undertaking 60 minutes or more of MVPA on fewer than seven days in the last week). Another key rationale for tracking physical activity trends in young people (between the ages of 9 to 18) is because high levels of physical activity in the childhood years is a cardinal predictor of high levels of physical activity in adulthood. ${ }^{32}$ Telema and colleagues ${ }^{33}$ concluded from their 21-year follow up study in young Finnish people that it is important to monitor school-age physical activity profiles as this appears to influence adult physical activity trends which ultimately reflect the public health of the general population.

The method of reporting levels of physical activity using self-report questionnaires is fraught with difficulty in young people. Measurement error may be inflated in 
young people due to issues of recall. Further, the recall instruments are likely to only pick up discrete bouts of activity that the young person is able to remember and may miss shorter, less defined bouts of activity. ${ }^{34}$ It is noteworthy that the recall questionnaire used in the current study (PAQ-A) was acknowledged by the Project Alpha investigators $^{34}$ as one of only three suitable recall questionnaires (along with Youth Risk Behaviour Surveillance Survey and Teen Health Survey) for use in population tracking of physical activity trends over time. It is also important to highlight that a systematic review of PA monitoring found $72 \%$ of self-reported measures provided over-estimates of children's PA when compared with objective measures, irrespective of sex. ${ }^{33}$

Declines in children's fitness ${ }^{6}$ have prompted calls for the introduction fitness testing, ${ }^{36}$ possibly as an addition to current measurement of BMI within the National Child Measurement Programme (NCMP]. ${ }^{37}$ These calls have met with resistance, warning of the negative impact fitness testing may have on "low ability and overweight children'. ${ }^{38}$ The assumption that overweight children will perform badly is false if fitness testing comprises assessments of muscular fitness; such statements serve to highlight the metonymy between 'fitness testing' and 'cardiorespiratory fitness testing' noted by Cohen and colleagues. ${ }^{39}$

Parents of children deemed overweight or obese according to their NCMP data receive a letter warning of potential ill effects to their child's health. Notwithstanding that this practice was and is not the unintended use for BMI, interpreting a single measure of body dimensions (form) in isolation in terms of health risk is clearly problematic. Based on BMI alone, one interpretation could be that SE boys and NE girls have greater health risk due to higher adiposity indicated by their higher BMIs. When BMI values are interpreted in conjunction with measures of muscular fitness (function) however, the data suggest no increased health risk as SE boys and NE girls are also stronger. The higher BMI values are likely to indicate greater lean body mass in these groups not excess adiposity. Furthermore, better muscular fitness is itself associated with better metabolic health, independent of BMI. A starting point toward systematic health surveillance beyond BMI could be the addition of a simple, objective assessment of muscular fitness such as handgrip strength. The practicability of handgrip strength has been 
demonstrated through its adoption as a measure of physical function in national surveys such as "Understanding Society-The UK Household Longitudinal Study". 40

The identification of significant regional variation in muscular fitness that were not explained by anthropometric differences suggests current normative data, derived from regional samples may need expanding and updating prior to roll-out of any national fitness surveillance programme. ${ }^{1,6}$

Study Limitations

We attempted to ensure that all the young people in the study completed the testing battery using the same protocols and equipment. The investigators attempted to ensure that each participant was appropriately motivated to achieve their best performance on the testing day, however, ensuring levels of participant motivation presents its own challenges. Each child was randomly selected to participate and we tried to ensure that our findings are generalisable to a wider population. However, we cannot be certain that the muscular performance and physical activity profiles from the children from the four schools selected $(2 x N E$; $2 \times \mathrm{SE}$ ) in our study are reflective of a broader population of age- and sex-matched children from England. We did not quantify the prevalence of active transportation. The PAQ-A provides information on the types of structured physical activity children engage in through sport or after-school clubs. It cannot, however, accurately quantify the volume of such activities, nor is it sensitive enough to pick up lighter-intensity or incidental bouts of physical activity. The findings of this study specifically relating to the role of physical activity should, therefore be interpreted with caution.

We did not control for biological maturation, therefore, even though we matched our cohort based on chronological age, it is possible that some schoolchildren were at different stages of biological maturation. It is well established that boys and girls mature at different stages; girls on average have their adolescent growth spurt two years earlier than boys and differences in muscular fitness may have been influenced by regional variations in timing of maturation. 
In conclusion, we found evidence of regional variation in physical activity muscular fitness between schoolchildren from northern and southern regions of England. These sex-specific differences in strength and power remained significant when scaled for body mass and could not be explained by accompanying variations in anthropometric measures. The systematic surveillance of children's physical activity and physical fitness throughout England is warranted in order to identify these regional inequalities on a larger scale.

\section{References}

1. Cohen, D.D, Voss, C., Taylor, M.J., Stasinopoulos, D.M., Delextrat. A., Sandercock. G.R. (2010). Handgrip strength in English schoolchildren. Acta Paediatrica, 99,1065-72.

2. Cohen, D.D., Voss, C., Taylor, M.J, Delextrat, A., Ogunleye, A.A., Sandercock, G.R. (2011). Ten-year secular changes in muscular fitness in English children. Acta Paediatrica, 100, e175-7.

3. Inskip, H.M., Godfrey, K.M., Martin, H.J., Simmonds, S.J., Cooper, C., Sayer, A.A. (2007). Size at birth and its relation to muscle strength in young adult women. Journal of Internal Medicine, 262, 368-74.

4. Tremblay, M.S., Shields, M., Laviolette, M., Craig, C.L., Janssen, I., Gorber, S.C. (2010). Fitness of Canadian children and youth: results from the 2007-2009 Canadian Health Measures Survey. Health Reports, 21, 7-20.

5. Moliner-Urdiales, D., Ruiz, J.R., Ortega, F.B., Jimenez-Pavon, D., VicenteRodriguez, G., Rey-Lopez, J.P.,... Moreno, L.A. (2010). Secular trends in healthrelated physical fitness in Spanish adolescents: The AVENA and HELENA Studies. Journal of Science and Medicine in Sport, 13, 584-8. 
6. Taylor, M.J., Cohen, D., Voss, C., Sandercock, G.R. (2010). Vertical jumping and leg power normative data for English school children aged 10-15 years. Journal of Sports Science, 28, 867-72.

7. Tomkinson, G.R. Global changes in anaerobic fitness test performance of children and adolescents (1958-2003). (2007). Scandinavian Journal of Medicine and Science in Sports, 17, 497-507.

8. Malina, R.M., Katzmarzyk, P.T. (2006). Physical activity and fitness in an international growth standard for preadolescent and adolescent children. Food and Nutrition Bulletin, 27, S295-313.

9. Colley, R.C., Garriguet, D., Janssen, I., Craig, C.L., Clarke, J., Tremblay, M.S. (2011). Physical activity of Canadian children and youth: accelerometer results from the 2007 to 2009 Canadian Health Measures Survey. Health Reports, 22, 1-9.

10. Andersen, L.B., Riddoch, C., Kriemler, S., Hills, A. (2011). Physical activity and cardiovascular risk factors in children. British Journal of Sports Medicine, 45, 8716.

11. Boreham, C.A.G., McKay, H.A. (2011). Physical activity in childhood and bone health. British Journal of Sports Medicine, 45, 877-9.

12. Warren, J.M., Ekelund, U., Besson, H., Mezzani, A., Geladas, N., Vanhees. L. (2010). Assessment of physical activity-a review of methodologies with reference to epidemiological research: a report of the exercise physiology section of the European Association of Cardiovascular Prevention and Rehabilitation. European Journal of Cardiovascular Prevention and Rehabilitation, 17, 127-39.

13. Welk, G.J., Corbin, C.B., Dale, D. (2010). Measurement issues in the assessment of physical activity in children. Research Quarterly in Exercise and Sport, 71, S59-73.

14. Corder, K., van Sluijs, E.M., Wright A., Whincup, P., Wareham, N.J., Ekelund, U. (2009). Is it possible to assess free-living physical activity and energy expenditure in young people by self-report? American Journal of Clinical Nutrition, $89,862-70$.

15. Voss, C., Ogunleye, A.A., Sandercock, G.S. (2013). Physical Activity Questionnaire for children and adolescents: English norms and cut-off points. Pediatrics International, 55, 498-507.

16. Boddy, L.M., Fairclough, S.J., Atkinson G, Stratton, G. (2012). Changes in Cardiorespiratory Fitness in 9-to 10.9-Year-Old Children: SportsLinx 1998-2010. Medicine and Science in Sports and Exercise 44, 481-486.

17. Boyle, SA. (2014). Comparison of Children's Fitness in the Northwest and Southwest of England. Advances in Physical Education, 4, 165-174.

18. World Medical Association Declaration of Helsinki: ethical principles for medical research involving human subjects. (2013). Journal of the American Medical Association, 310, 2191-4. 
19. Urban and Rural Classification. www.gov.uk/government/statistics/2011-ruralurban-classification [accessed: 15th October 2015].

20. Cole, T.J., Bellizzi, M.C., Flegal, K.M., Dietz, W.H. (2000). Establishing a standard definition for child overweight and obesity worldwide: international survey. British Medical Journal, 320, 1240-3.

21. Department for Communities and Local Government. The English Indices of Deprivation 2007. Summary. Communities and Local Government Publications, Wetherby, UK, 2007.

22. Sayers, S. P., Harackiewicz, D. V., Harman, E. A., Frykman, P. N., Rosenstein, M.T. (1999). Cross-validation of three jump power equations. Medicine and Science in Sports and Exercise, 31, 572-577.

23. Hager-Ross, C., Rosblad, B. (2002). Norms for grip strength in children aged 416 years. Acta Paediatrica, 91, 617-25.

24. Ortega, F.B., Artero, E.G., Ruiz, J.R., Vicente-Rodriguez, G., Bergman, P., Hagstromer, M. , ... Castillo, M.J; HELENA Study Group. (2008). Reliability of healthrelated physical fitness tests in European adolescents. International Journal of Obesity, 32, S49-57.

25. Espana-Romero, V., Ortega, F.B., Vicente-Rodriguez, G., Artero, E.G., Rey, P.J., Ruiz. J.R. (2010). Elbow position affects handgrip strength in adolescents: validity and reliability of Jamar, DynEx, and TKK dynamometers. Journal of Strength \& Conditioning Research, 24, 272-7.

26. Voss, C., Ogunleye, A.A., Sandercock, G.R. (2013). Physical activity questionnaire for children and adolescents: English norms and cut-points. Pediatrics International, 55, 498-507.

27. Dumith, S.C., Ramires, V.V., Souza, M.A., Moraes, D.S., Petry, F.G., Oliveira, E.S., Hallal, P.C. (2010). Overweight/Obesity and Physical Fitness among Children and Adolescents. Journal of Physical Activity and Health, 7, 641-648.

28. Gonzalez-Suarez, C.B., Grimmer-Somers, K. (2011). The Association of Physical Activity and Physical Fitness with Pre-Adolescent Obesity: An Observational Study in Metromanila, Philippines. Journal of Physical Activity and Health, 8, 804-810.

29. Tremblay, M.S., Williams, J.D. (2003). Is the Canadian Childhood Obesity Epidemic Related to Physical Inactivity? International Journal of Obesity, 27, 11001105.

30. Parson, T.J., Power, C., Manor, O. (2005). Physical Activity, Television Viewing and Body Mass Index: A Cross-Sectional Analysis from Childhood to Adulthood in the 1958 British Cohort. International Journal of Obesity, 29, 1212-1221.

31. Boyle, S.E., Walters, S.J., Jones, G.L. (2010). Physical Activity, Quality of Life, Weight Status and Diet in Adolescents. Quality of Life Research, 19, 943-954.

32. Health Survey for England 2012. Volume 1: Health, social care and lifestyle. Leeds: The NHS Information Centre for health and social care; 2013. 
33. Telema, R., Yang, X., Viikari, J., Valimaki, I., Wanne, O., Raitakari, O. (2005). Physical activity from childhood to adulthood - A 21-year tracking study. American Journal of Preventive Medicine, 28, 267-273.

34. Biddle, S.J.H., Gorely, T., Pearson, N., Bull, F.C. (2011). An assessment of self-reported physical activity instruments in young people for population surveillance: Project ALPHA. International Journal of Behavioural Nutrition and Physical Activity, 8, 1.

35. Adamo, K.B., Prince, S.A., Tricco, A.C., Connor-Gorber. S., Tremblay, M. (2009). A comparison of indirect versus direct measures for assessing physical activity in the pediatric population: a systematic review. International Journal of Pediatric Obesity, 4, 2-27.

36. Donaldson L. The 2009 Annual Report of the Chief Medical Officer. (2010). http://webarchive.nationalarchives.gov.uk/20130107105354/http://www.dh.gov. uk/prod_consum_dh/groups/dh_digitalassets/@dh/@en/@ps/documents/digitalass et/dh_114012.pdf. [Accessed 16 October 2015].

37. UK Active 2015- Generation Inactive. http://www.ukactive.com/downloads/managed/ON02629_UK_Active_Kids_report_ online_spreads_FP.PDF. [Accessed 16 October 2015].

38. Cale, L., Harris, J., Chen, M.H. (2007). More Than 10 Years After "The Horse is Dead ... ." Surely It Must Be Time to "Dismount"?! Pediatric Exercise Science, 19, 115-131.

39. Cohen, D.D., Voss, C., Sandercock, G,R. (2015). Fitness Testing for Children: Let's Mount the Zebra! Journal of Physical Activity for Health, 12, 597-603.

40. Understanding Society https://www.understandingsociety.ac.uk/ [Accessed $31^{\text {st }}$ March 2016]. 
Table I. Raw scores for anthropometry, hand-grip strength, vertical jump test performance, and physical activity profiles in boys and girls from North-Eastern (NE) and South-Eastern (SE) regions of England

\begin{tabular}{|c|c|c|c|c|c|}
\hline \multirow[b]{2}{*}{ Boys } & \multicolumn{2}{|c|}{ SE } & \multicolumn{2}{|c|}{ NE } & \\
\hline & Mean & SD & Mean & SD & $P$-value \\
\hline Age (years) & 12.6 & 1.26 & 13.0 & 1.44 & $<0.001$ \\
\hline Stature $(\mathrm{m})$ & 1.59 & 1.17 & 1.58 & 1.16 & 0.34 \\
\hline Mass (kg) & 50.4 & 13.0 & 49.1 & 11.8 & 0.19 \\
\hline $\mathrm{BMI}$ & 19.0 & 3.62 & 19.8 & 5.17 & 0.98 \\
\hline Underweight & $6.7 \%$ & & $14.9 \%$ & & 0.04 \\
\hline Normal weight & $67.8 \%$ & & $62.4 \%$ & & $<0.001$ \\
\hline Overweight & $20.6 \%$ & & $13.7 \%$ & & $<0.001$ \\
\hline Obese & $5.0 \%$ & & $7.1 \%$ & & 0.99 \\
\hline Handgrip strength (kg) & 26.3 & 7.61 & 22.8 & 5.92 & $<0.001$ \\
\hline VJT (m) & 0.33 & 0.23 & 0.28 & 0.13 & $<0.001$ \\
\hline PAQ-A (1-5) & 2.91 & 0.72 & 2.51 & 0.76 & $<0.001$ \\
\hline Girls & $\mathrm{SE}$ & & $\mathrm{N}$ & & $P$-value \\
\hline Age & 12.6 & 1.30 & 13.1 & 1.43 & $<0.001$ \\
\hline Stature $(\mathrm{m})$ & 1.57 & 0.92 & 1.63 & 1.32 & $<0.001$ \\
\hline Mass (kg) & 50.9 & 12.3 & 53.6 & 15.6 & 0.02 \\
\hline BMI & 20.3 & 4.74 & 19.9 & 4.40 & 0.22 \\
\hline Underweight & $7.8 \%$ & & $15.2 \%$ & 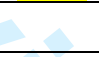 & $<0.001$ \\
\hline Normal weight & $57.8 \%$ & & $63.6 \%$ & $P$ & 0.08 \\
\hline Overweight & $17.5 \%$ & & $14.0 \%$ & 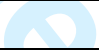 & 0.22 \\
\hline Obese & $5.8 \%$ & & $7.2 \%$ & +8 & 0.40 \\
\hline Handgrip strength (kg) & 22.4 & 5.71 & 27.9 & 9.76 & $<0.001$ \\
\hline VJT $(m)$ & 0.28 & 0.33 & 0.30 & 0.14 & $<0.01$ \\
\hline PAQ-A (1-5) & 2.56 & 0.59 & 2.57 & 0.81 & 0.81 \\
\hline
\end{tabular}


Table II. Standardised scores for anthropometric and performance variables, and physical activity profiles in the boys and girls in the NE and SE regions of England

\begin{tabular}{|c|c|c|c|c|}
\hline \multicolumn{3}{|c|}{ Region } & Mean & Std. Deviation \\
\hline \multirow{15}{*}{ Boys } & \multirow{3}{*}{ Stature_SDS } & & & \\
\hline & & SE & 0.83 & 1.27 \\
\hline & & NE & 0.93 & 1.37 \\
\hline & \multirow{2}{*}{ Weight_SDS } & SE & 0.82 & 1.06 \\
\hline & & $\mathrm{NE}$ & 0.77 & 1.13 \\
\hline & \multirow[t]{2}{*}{ BMI_SDS } & SE & 0.55 & 1.33 \\
\hline & & $\mathrm{NE}$ & 0.34 & 1.43 \\
\hline & \multirow[t]{2}{*}{ HG_SDS } & SE & 0.10 & 1.01 \\
\hline & & $\mathrm{NE}$ & -0.10 & 1.05 \\
\hline & \multirow[t]{2}{*}{ VJT_SDS } & SE & 0.25 & 1.01 \\
\hline & & $\mathrm{NE}$ & -0.56 & 1.58 \\
\hline & \multirow[t]{2}{*}{ Peak Power_SDS } & SE & 0.013 & 0.96 \\
\hline & & NE & -0.53 & 1.31 \\
\hline & \multirow[t]{2}{*}{ PAQ-A_SDS } & SE & 0.023 & 0.86 \\
\hline & & $\mathrm{NE}$ & -0.32 & 0.92 \\
\hline \multirow[t]{14}{*}{ Girls } & \multirow[t]{2}{*}{ Stature_SDS } & SE & 0.39 & 1.13 \\
\hline & & $\mathrm{NE}$ & 0.54 & 1.85 \\
\hline & \multirow[t]{2}{*}{ Weight_SDS } & SE & 0.64 & 1.18 \\
\hline & & $\mathrm{NE}$ & 0.36 & 1.11 \\
\hline & \multirow[t]{2}{*}{ BMI_SDS } & SE & 0.52 & 1.35 \\
\hline & & NE & 0.02 & 1.51 \\
\hline & \multirow[t]{2}{*}{ HG_SDS } & SE & 0.15 & 0.91 \\
\hline & & NE & -0.10 & 1.02 \\
\hline & \multirow[t]{2}{*}{ VJT_SDS } & SE & 0.21 & 1.14 \\
\hline & & NE & -0.12 & 1.63 \\
\hline & \multirow[t]{2}{*}{ Peak Power_SDS } & SE & 0.13 & 0.89 \\
\hline & & NE & -0.45 & 1.27 \\
\hline & \multirow[t]{2}{*}{ PAQ-A_SDS } & SE & -0.14 & 0.84 \\
\hline & & $\mathrm{NE}$ & -0.06 & 1.03 \\
\hline
\end{tabular}

$\mathrm{VJT}=$ vertical jump test; $\mathrm{HG}=$ hand-grip strength; $\mathrm{SDS}=$ standard deviation score; $\mathrm{PAQ}=$ physical activity profile; $\mathrm{BMI}=$ body mass index; $\mathrm{SE}=$ south east; $\mathrm{NE}=$ north east. 
Table III. Differences in standardised physical activity profiles and selected fitness scores between boys and girls in the NE and SE regions of England

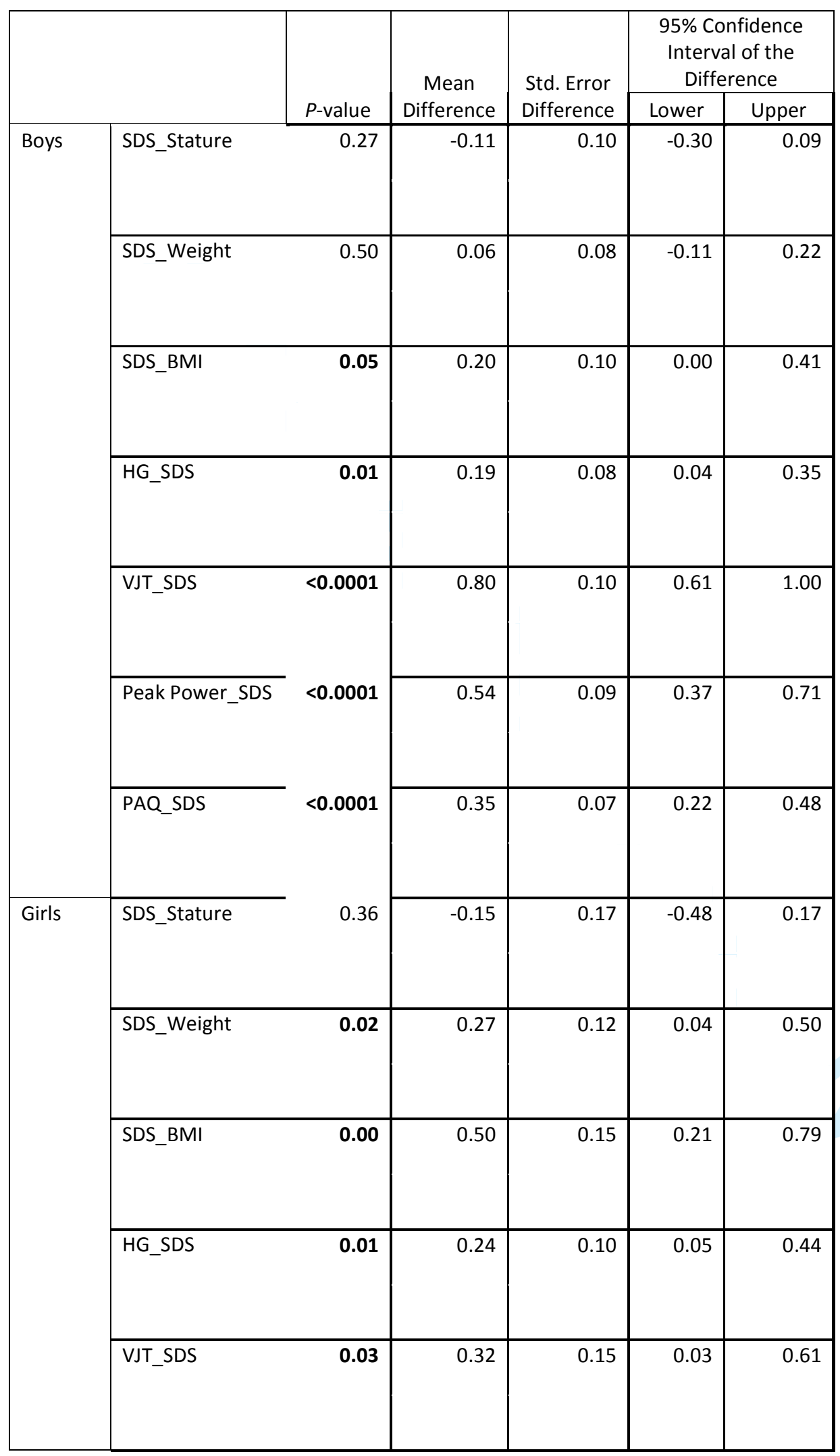

URL: http:/mc.manuscriptcentral.com/tejs 
1

2

3

4

5

6

7

8

9

10

11

12

13

14

15

16

17

18

19

20

21

22

23

24

25

26

27

28

29

30

31

32

33

34

35

36

37

38

39

40

41

42

43

44

45

46

47

48

49

50

51

52

53

54

55

56

57

58

59

60

\begin{tabular}{|l|l|r|r|r|r|} 
& Peak Power_SDS $<0.0001$ & 0.59 & 0.12 & 0.36 & 0.81 \\
& & & & & \\
\hline PAQ_SDS & 0.43 & -0.08 & 0.10 & -0.27 & 0.11 \\
\hline
\end{tabular}

VJT= vertical jump test; $\mathrm{HG}=$ hand-grip strength; $\mathrm{SDS}=$ standard deviation score; $\mathrm{PAQ}=$ physical activity profile; $\mathrm{BMI}=$ body mass index. 
Table IV. Hierarchical regression analysis showing predictors of hand-grip strength and peak power in boys and girls from the NE and SE of England (adjusted for age, BMI, physical activity profiles and area level deprivation)

\begin{tabular}{|c|c|c|c|c|c|}
\hline Handgrip (kg) & Boys & & Girls & & \\
\hline Model $^{1}$ & $\beta$ & $(95 \% \mathrm{Cl})$ & & $\beta$ & $(95 \% \mathrm{Cl})$ \\
\hline Location (NE=1) & -3.91 & $(-4.70 \text { to }-2.93)^{* * *}$ & Location (NE=1) & 2.00 & (0.91 to 3.12$)^{* * *}$ \\
\hline Age & 3.22 & $(2.82 \text { to } 3.61)^{* * *}$ & Age & 3.40 & $(2.89 \text { to } 3.71)^{* * *}$ \\
\hline Height & 1.41 & $(1.07 \text { to } 1.62)^{* * *}$ & Height & 1.50 & $(1.13 \text { to } 3.02)^{* * *}$ \\
\hline BMI & 1.22 & $(0.93 \text { to } 1.53)^{* *}$ & BMI & 0.70 & $(0.31 \text { to } 1.22)^{* *}$ \\
\hline Model $^{2}$ & & & Model $^{2}$ & & \\
\hline Location (NE =1) & -3.52 & $(-4.42 \text { to }-2.82)^{* * *}$ & Location (NE =1) & 1.90 & $(0.81 \text { to } 3.02)^{* *}$ \\
\hline Age & 3.23 & $(2.82 \text { to } 3.62)^{* * *}$ & Age & 3.40 & (3.02 to 3.44$)^{* * *}$ \\
\hline Height & 1.32 & (1.01 to 1.63$)^{* * *}$ & Height & 1.60 & (1.32 to 1.92$)^{* * *}$ \\
\hline BMI & 1.22 & (0.91 to 1.53$)^{* * *}$ & BMI & 1.20 & (0.9 1 to 1.53 ) *** \\
\hline Physical Activity & 0.97 & $(0.50 \text { to } 1.51)^{* * *}$ & Physical Activity & 0.90 & (0.43 to 1.41$)^{* *}$ \\
\hline Model $^{3}$ & & & Model $^{3}$ & & \\
\hline Location (NE =1) & -1.50 & $(-3.3$ to 0.3$)$ & Location (NE =1) & 2.00 & (0.1 to 3.9$)^{*}$ \\
\hline Age & 3.25 & $(2.8$ to 3.6$) * * *$ & Age & 3.40 & (3.0 to 3.9$) * * *$ \\
\hline Height & 1.38 & (1.0 to 1.6$) * * *$ & Height & 1.50 & (1.1 to 1.8$)^{* * *}$ \\
\hline BMI & 1.18 & (0.9 to 1.5$)^{* * *}$ & BMI & 0.70 & (0.3 to 1.1$)^{* * *}$ \\
\hline Physical Activity & 0.96 & $(0.5 \text { to } 1.5)^{* * *}$ & Physical Activity & 0.93 & $(0.4 \text { to } 1.4)^{* * *}$ \\
\hline Deprivation & 0.72 & $(0.1$ to 1.4$) *$ & Deprivation & 0.04 & (-0.6 to 0.7$)$ \\
\hline Handgrip (kg/kgBW) & Boys & 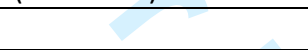 & Girls & & \\
\hline Model $^{1}$ & $\beta$ & $(95 \% \mathrm{Cl})$ & & & \\
\hline Location ( $\mathrm{NE}=1$ ) & -0.01 & $(-0.05$ to 0.02$)$ & Location (NE=1) & 0.30 & (0.1 to 0.4$)^{*}$ \\
\hline Age & 0.20 & (0.1 to 0.22 ) & Age & 0.20 & $(0.1 \text { to } 0.3)^{*}$ \\
\hline BMI & -0.20 & $(-0.2)$ & BMI & -0.50 & $(-0.7 \text { to }-0.4)^{* *}$ \\
\hline Model $^{2}$ & & & Model $^{2}$ & & \\
\hline Location (NE =1) & -0.67 & $(-0.85 \text { to }-0.48)^{* * *}$ & Location (NE =1) & 0.30 & (0.1 to 0.4$)^{*}$ \\
\hline Age & 0.02 & $(0.009 \text { to } 0.022)^{* * *}$ & Age & 0.20 & (0.1 to 0.3$)^{*}$ \\
\hline BMI & -0.35 & $(-0.41 \text { to }-0.29)^{* * *}$ & BMI & -0.60 & $(-0.1 \text { to }-0.6)^{* *}$ \\
\hline Physical Activity & 0.20 & $(0.10 \text { to } 0.30)^{* * *}$ & Physical Activity & 0.2 & $(-0.1 \text { to }-0.3)^{* *}$ \\
\hline Model $^{3}$ & & & \begin{tabular}{|l|} 
Model $^{3}$ \\
\end{tabular} & & \\
\hline Location (NE =1) & -0.01 & (-0.49 to 0.38$)$ & Location (NE=1) & 0.30 & (0.1 to 0.4$)^{*}$ \\
\hline Age & 0.16 & $(0.09 \text { to } 0.22)^{* * *}$ & Age & 0.20 & (0.1 to 0.3$)^{*}$ \\
\hline $\mathrm{BMI}$ & 1.38 & $(-0.42 \text { to- } 0.29)^{* * *}$ & BMI & -0.60 & $(-0.1 \text { to }-0.6)^{* *}$ \\
\hline Physical Activity & 0.20 & $(0.10 \text { to } 0.30)^{* * *}$ & Physical Activity & 0.20 & $(-0.1 \text { to }-0.3)^{* *}$ \\
\hline Deprivation & 0.21 & (0.01 to 0.33$)^{*}$ & Deprivation & 0.04 & $(-0.08$ to 0.16$)$ \\
\hline \multicolumn{6}{|l|}{ Peak Power (W) } \\
\hline Model $^{1}$ & $\beta$ & $(95 \% \mathrm{Cl})$ & & $\beta$ & $(95 \% \mathrm{Cl})$ \\
\hline Location (NE=1) & -415.30 & $(-51.1$ to 298.0$)$ & Location (NE=1) & 174.40 & (51.7 to 297.2)* \\
\hline Age & 332.10 & (297.6 to 366.6 ) & Age & 307.30 & (265 1 to 360.1$)^{*}$ \\
\hline \multirow[t]{2}{*}{$\mathrm{BMI}$} & 235.70 & (203.5 to 267.9) & BMI & 221.20 & (177 to 265.3$)^{* *}$ \\
\hline & & $R^{2}=0.53$ & & & $R^{2}=0.37$ \\
\hline Model $^{2}$ & $\beta$ & $(95 \% \mathrm{Cl})$ & Model $^{2}$ & B & $(95 \% \mathrm{Cl})$ \\
\hline Location (NE =1) & -372.30 & $(-469.1 \text { to } 276.6)^{* *}$ & Location (NE=1) & 170.70 & $(46.7 \text { to } 294.1)^{* *}$ \\
\hline Age & 335.10 & $(297.6 \text { to } 366.6)^{* * *}$ & Age & 309.10 & (265.6 to 360.1$)^{*}$ \\
\hline $\mathrm{BMI}$ & 240.10 & $(203.5 \text { to } 267.9)^{* * *}$ & BMI & 222.00 & $(178.3 \text { to } 266.3)^{* *}$ \\
\hline Physical Activity & 103.40 & $(50.3 \text { to } 156.1)^{* * *}$ & Physical Activity & 22.90 & $(-34.5$ to 89.3$)$ \\
\hline Model $^{3}$ & & $R^{2}=0.54$ & Model $^{3}$ & & $\mathrm{R}^{2}=0.37$ \\
\hline Location ( $\mathrm{NE}=1$ ) & -448.20 & $(-649.2 \text { to }-247.3)^{* * *}$ & Location (NE=1) & 116.0 & $(-333.7$ to 101.7$)$ \\
\hline Age & 335.10 & $(300.7 .6 \text { to } 369.3)^{* * *}$ & Age & 309.1 & (303.6 to 360.1$)^{*}$ \\
\hline BMI & 241.50 & (203.5 to 267.9$)^{* * *}$ & BMI & 230.9 & () 186.3 to 275.4$)^{* *}$ \\
\hline Physical Activity & 103.40 & $(50.3 \text { to } 156.8)^{* * *}$ & Physical Activity & 21.60 & $(-35.7$ to 78.2$)$ \\
\hline
\end{tabular}

URL: http:/mc.manuscriptcentral.com/tejs 


\begin{tabular}{|c|c|c|c|c|c|}
\hline Deprivation & -27.70 & (-32.1 to 67.7$)$ & Deprivation & -114.50 & $(-186.1 \text { to }-43.0)^{*}$ \\
\hline & & $R^{2}=0.55$ & & & $R^{2}=0.38$ \\
\hline \multicolumn{6}{|c|}{$\begin{array}{l}\text { Peak Power (W/kg } \\
\text { BW) }\end{array}$} \\
\hline Model $^{1}$ & $\beta$ & $(95 \% \mathrm{Cl})$ & & $\beta$ & $(95 \% \mathrm{Cl})$ \\
\hline Location (NE=1) & -7.28 & $(-9.21$ to -5.36$)$ & Location ( $\mathrm{NE}=1$ ) & -0.82 & $(-1.18$ to 19.9$)$ \\
\hline Age & 3.60 & (2.88 to 4.29 ) & Age & 0.20 & $(-3.31 \text { to } 1.63)^{* * *}$ \\
\hline \multirow[t]{2}{*}{ BMI } & 0.59 & (0.06 to 1.24 ) & BMI & -1.23 & $(-2.10 \text { to }-0.34)^{*}$ \\
\hline & & $R^{2}=0.19$ & & & $R^{2}=0.059$ \\
\hline Model $^{2}$ & $\beta$ & $(95 \% \mathrm{Cl})$ & Model $^{2}$ & $\beta$ & $(95 \% \mathrm{Cl})$ \\
\hline Location (NE =1) & -6.52 & $(-8.48 \text { to }-4.45)^{* * *}$ & Location (NE =1) & -0.88 & $(-3.3 \text { to } 1.88)^{*}$ \\
\hline Age & 3.64 & $(2.94 \text { to } 4.34)^{* * *}$ & Age & 2.39 & $(1.54 \text { to } 3.23)^{* * *}$ \\
\hline BMI & 0.66 & (0.01 to 2.32$)^{*}$ & BMI & -1.21 & $(-2.10 \text { to }-0.33)^{* *}$ \\
\hline Physical Activity & 1.77 & $(0.68 \text { to } 2.77)^{* *}$ & Physical Activity & 0.36 & (0.83 to 1.50$)$ \\
\hline \multirow[t]{2}{*}{ Model $^{3}$} & & $R^{2}=0.21$ & & & $R^{2}=0.061$ \\
\hline & $\beta$ & $(95 \% \mathrm{Cl})$ & Model $^{3}$ & & $(95 \% \mathrm{Cl})$ \\
\hline Location (NE =1) & -5.08 & $(-9.19 \text { to }-0.97)^{*}$ & Location (NE =1) & -3.92 & $(-8,27$ to 0.43$)$ \\
\hline Age & 3.65 & $(2.95 \text { to } 4.35)^{* * *}$ & Age & 2.33 & (1.48 to 3.18$)^{*}$ \\
\hline BMI & 0.65 & $(-0.01$ to 1.30$)$ & BMI & 1.12 & $(-2.07 \text { to }-0.23)^{*}$ \\
\hline Physical Activity & 1.78 & $(0.69 \text { to } 2.80)^{* *}$ & Physical Activity & 0.35 & (-0.79 to 1.48$)$ \\
\hline \multirow{2}{*}{ Deprivation } & 0.52 & $(-0.79 \text { to } 1.84)^{*}$ & Deprivation & -1.21 & (-2.64 to 0.22$)$ \\
\hline & & $R^{2}=0.22$ & & & $R^{2}=0.064$ \\
\hline
\end{tabular}

${ }^{1}$ Association between location and muscular fitness adjusted for age and BMI

${ }^{2}$ Adjusted for age, BMI, and physical activity

${ }^{3}$ Fully adjusted model; adjusted for age, BMI, physical activity, and area level deprivation.

${ }^{*} P<0.05,{ }^{* *} P<0.01,{ }^{* * *} P<0.001$ 